of spontaneous circulation in experimental group. On seventh day after CPR, neurons apoptosis was examined using terminal deoxynucleotidyl transferase mediated dUTP biotin nick end labelling (TUNEL) staining and the expression of caspase-3 was detected by the immunohistochemical strepto avidin biotinperoxidase complex (SABC) method in cortex, hippocampus CA1 region and cerebellum of the rats.

Results 1 . There were 12 and 10 rats completed the experiment in the experimental and control group respectively. Their fate between the two groups was no significant difference $\left(\chi^{2}=0.404, p=0.376\right) .2$. On seventh day after CPR, The serum concentrations of $\mathrm{H}_{2} \mathrm{~S}$ was $9.12 \pm 3.17 \mu \mathrm{mol} / 1$ in the experimental group and the contrast was $3.72 \pm 1.05 \mu \mathrm{mol} / 1$, the difference between the two groups had statistic significance $(t=5.136, p=0.000)$. 3. Compared with the control group, the experimental group's neurons apoptosis index and the sum of integrated optical density (IOD) of caspase- 3 in cortex, hippocampus CA1 region and cerebellum were obviously reduced $(\mathrm{p}<0.05)$.

Conclusion After CPR, $\mathrm{H}_{2} \mathrm{~S}$ can inhabit neurons apoptosis and its mechanism may be through caspase- 3 pathway. It may play a role in the treatment of the brain injury after CA.

\section{e0005 MODEL OF CARDIAC ARREST IN RATS BY TRANSCUTANEOUS ELECTRICAL EPICARDIUM STIMULATION}

doi:10.1136/hrt.2010.208967.5

Lin Ji-Yan, Li Xin, Li Hui, Wei Hong-Yan, Liu Rong, Hu Chun-Lin, Dai Gang, Liao Xiao-Xing. The First Affiliated Hospital of Sun Yat-sen University

Objective To establish a new model of Cardiac Arrest (CA) in rats by transcutaneous electrical epicardium stimulation.

Methods Two acupuncture needles connected to the anode and cathode of a stimulator were transcutaneously inserted into the epicardium as electrodes. The stimulating current was steered to the epicardium and the stimulation was maintained for 3 min to induce CA. Cardiopulmonary resuscitation (CPR) was performed at 6 min after a period of nonintervention.

Results The success rate of induction was $12 / 20$ at the current intensity of $1 \mathrm{~mA}$; and reached 20/20 when the current intensity was increased to $2 \mathrm{~mA}$. The average time from the electrical stimulation to CA induction was $5.10( \pm 2.81) \mathrm{s}$. When the electrical stimulation stopped, $18 / 20$ rats had ventricular fibrillation and 2/20 rats had pulseless electrical activity. CPR was performed for averagely $207.4( \pm 148.8) \mathrm{s}$. The restoration of spontaneous circulation was $20 / 20$. The death rate within $4 \mathrm{~h}$ after CA was $5 / 20$, and the 72 -h survival rate was $10 / 20$. There were only two cases of complications, a minor muscle contraction and a minor lung lobe injury.

Conclusion The model of CA in rats induced by transcutaneous electrical epicardium stimulation is a stable model that requires lowintensity current and has fewer complications.

\section{e0006 EFFECTS OF NEOTYPE PERITONEAL COOLING ON THE INJURED OF INTESTINAL MUCOUS AFTER CARDIOPULIMONARY RESUSCITATION IN RABBITS}

doi:10.1136/hrt.2010.208967.6

Dai Xuan, Liao Xiao-Xing, Hu Chun-Lin, Wei Hong-Yan, Li Xin, Zhan Hong. The First Affiliated Hospital of Sun Yat-Sen University

Objective To explore whether the peritoneal cooling after cardiopulmonary resuscitation could improve the injured of intestinal mucous in rabbits.

Methods 36 adult New Zealand rabbits were induced ventricular fibrillation by $\mathrm{AC}$ current. After the restore of spontaneous circulation (ROSC), rabbits were randomly divided into three groups according to the way of body temperature controlling, that is, nomothermia group (NT), surface cooling group (SC) and peritoneal cooling group (PC). The changing of tympanic temperature and peritoneal temperature were observed after ROSC. The animals were sacrificed by over anaesthesia after ROSC for $12 \mathrm{~h}$, the end ileum was removed and fixed in formalin, the histological injured and the expression of TNF-a and VCAM-1 in ileum were observed by H.E staining and immune chemical methods.

Results 12 animals in each group, 9 in group NT, 10 in group SC and 9 in group PC were successfully resuscitated; all animals were on mechanical ventilation for 2 to 4 h. 5, 6 and 8 animals in each group respectively survived to the end of the experiment. The temperatures of tympanic and peritoneal cavity of animals in group NT were maintained in normal range. The tympanic temperature of animals in group SC and PC was arrived target temperatures at $29 \pm 6.55 \mathrm{~min}$ and $62 \pm 8.27 \mathrm{~min}$. During the stage of maintenance of hypothermia, the tympanic and peritoneal temperatures of animals in group SC were in range 33 to $35^{\circ} \mathrm{C}$, while the peritoneal temperatures of animals in group $\mathrm{PC}$ were in range 31 to $34^{\circ} \mathrm{C}, 1$ to $2{ }^{\circ} \mathrm{C}$ lower than the tympanic temperature. The scores of histological injured of ileum were $1.43 \pm 0.53$ in group PC, $3.4 \pm 0.55$ in group NT and $3.17 \pm 0.41$ in group SC. The differences among them were significantly, PC versus SC, $p<0.000$; PC versus NT, $p<0.000$; while $\mathrm{SC}$ versus NT, $\mathrm{p}=0.30$. The expression of TNF-a in ileum was $9.98 \pm 1.79 \%$ in group NT, $5.87 \pm 1.43 \%$ in group SC and $3.78 \pm 0.53 \%$ in group PC, the differences among them were significantly. The phenomenon of the expression of VCAM-1 was little like the TNF-a, $3.78 \pm 0.53 \%$ in group PC was significantly from the $8.53 \pm 1.53 \%$ in group NT and $5.92 \pm 1.06 \%$ in group SC.

Conclusion The neotype peritoneal cooling can improve the injured of ileum mucous beside quickly induce hypothermia after ROSC in rabbits.

\section{e0007 THE EFFECT OF OMEPRAZOLE ON THE OXIDATIVE STRESS AND ACUTE ATRIAL ELECTRICAL REMODELLING IN RABBITS}

doi:10.1136/hrt.2010.208967.7

${ }^{1}$ Yuan Guan, ${ }^{2}$ Kai Ren, ${ }^{2}$ Dexian Zhang, ${ }^{2}$ Zhaoliang Shan, ${ }^{2}$ Yutang Wang, ${ }^{3}$ Yunming Li, ${ }^{2}$ Hongyang Guo, ${ }^{2}$ Ye Zhang. ${ }^{1}$ PLA General Hospital; ${ }^{2}$ General Hospital of PLA; ${ }^{3}$ General Hospital of Chengdu Military Region

Objective To investigate the effect of omeprazole on the acute atrial electrical remodelling and oxidative stress status in rabbit atrial fibrillation (AF) model.

Methods 18 rabbits were randomly divided into atrial tachypacing (ATP) group, sham operating (SM) group, and atrial tachypacing with omeprazole therapy $(\mathrm{A}+\mathrm{O})$ group. In the ATP group and $\mathrm{A}+\mathrm{O}$ group the right atrium was tachypaced at $500-600 \mathrm{bpm}$ to induce and maintain $\mathrm{AF}$ for $3 \mathrm{~h}$. The $\mathrm{A}+\mathrm{O}$ group were given intravenous administration of omeprazole treatment $(4 \mathrm{mg} / \mathrm{kg}) 15 \mathrm{~min}$ before tachypacing. The ATP group were given intravenous administration of physiological saline $10 \mathrm{ml} 15$ min before pacing. The SM group were not paced. The atrial electrophysiological indexes (AERP, Rate adaptive of AERP) were measured at different time point (baseline, $0.5 \mathrm{~h}, 1 \mathrm{~h}, 1.5 \mathrm{~h}, 2 \mathrm{~h}, 2.5 \mathrm{~h}$ and $3 \mathrm{~h}$ after pacing). Oxidative stress markers (SOD, MDA, T-NOS) in serum were measured at different time point (baseline and $3 \mathrm{~h}$ after pacing).

Results 1. Compare to SM group, the atrial effective refractory period (ERP) at a cycle length of $200 \mathrm{~ms}$ was decreased from $93.89 \pm 5.88$ to $72.78 \pm 5.37 \mathrm{~ms}(\mathrm{p}<0.01)$ after pacing in ATP group, and the Rate adaptive of ERP appeared non-performing significantly after tachypacing in ATP group (from $0.10 \pm 0.02$ to $0.04 \pm 0.01$, $\mathrm{p}<0.01$ ); but no change in $\mathrm{A}+\mathrm{O}$ group, with ERP and Rate adaptive of ERP averaging $100.17 \pm 8.93 \mathrm{~ms}$ and $0.09 \pm 0.02$. The level of lipid 